\section{Is there still a place for improvement in acute coronary syndrome risk stratification?}

\author{
Ana Teresa Timóteo
}

In ST-elevation myocardial infarction (STEMI), early diagnosis by the specific ECG changes and timely reperfusion therapy, has remarkably improved the outcome. The non-ST-elevation acute coronary syndrome (NSTEACS) cohort is usually larger and more heterogeneous. Given the wide spectrum of antithrombotic and antiplatelet therapies available today, as well as improvements in invasive management, it requires a timely management decision by physicians. Although these therapeutic options improve outcome, they can increase both the rate of complications and costs. For that reason, an informed decision is important and risk stratification scores are helpful tools for an early triage and adequate identification of intermediate/high-risk groups that can benefit from an invasive strategy.

In the last 15 years, several risk stratification scores for acute coronary syndromes (ACSs) have been developed. The first risk scores to be widely implemented in clinical practice were Thrombolysis in Myocardial Infarction risk score for STEMI and NSTEACS. ${ }^{12}$ These scores were developed from cohorts of patients included in randomised clinical trials. Subsequent risk scores used the same methodology. Global Registry of Acute Coronary Events (GRACE) risk score was published in 2003 by the GRACE registry group, initially for hospital mortality in patients with ACS and later on for 6-month mortality. ${ }^{3} 4$ This score was developed from a registry that represents real-world patients instead of the highly controlled and selected population found in clinical trials, which is considered a limitation of previous risk scores. Elderly patients and patients with severe renal dysfunction are usually excluded from clinical trials. This might explain the inclusion of new variables such as renal

\footnotetext{
${ }^{1}$ Santa Marta Hospital, CHLC, Lisbon, Portugal; ${ }^{2}$ NOVA Medical School, Lisbon, Portugal; ${ }^{3}$ Working Group on Intensive Cardiac Care of the Portuguese Society of Cardiology, Lisbon, Portugal
}

Correspondence to Professor Ana Teresa Timóteo, Hosp Santa Marta, Rua Santa Marta, Lisbon 1120, Portugal; ana_timoteo@yahoo.com function in the new real-life score. Nowadays, due to its high predictive accuracy for both short-term and medium-term all-cause mortality, it is the most widely used risk score in ACS context. Since then, many authors have shown the incremental prognostic value of other clinical and laboratorial variables on top of GRACE risk score.

Patients with chronic obstructive pulmonary disease (COPD) are at high-risk of experiencing ACS. ${ }^{5}$ This risk is usually attributed to common risk factors, such as higher age and smoking, as well as to the systemic inflammatory response present in COPD. ${ }^{6}$ A patient with COPD experiencing ACS have a subsequent increased risk of recurrent ischaemic events and long-term all-cause mortality, explained by the fact that they have higher prevalence of age-related and smokingrelated comorbidities and are less likely to receive invasive treatment and guidelinerecommended secondary prevention therapies, including a lower likelihood of cardiac rehabilitation attendance after myocardial infarction. ${ }^{5}$ The association with prolonged hypoxaemia and the use of inhaled $\beta$-agonists among patients with COPD could also contribute to the elevated arrhythmic risk, which potentially may also influence inhospital outcome. ${ }^{6}$ Also in patients that survive to discharge after and ACS, COPD has an important long-term prognostic impact. The longtErm follow-up of antithrombotic management Patterns In acute CORonary syndrome patients (EPICOR) study showed that COPD is present in 6\% of the patients that survived to hospital discharge after ACS and it is an independent predictor of all-cause 1-year mortality with a crude mortality rate of $8.8 \%$. However, the impact is only significant in the NSTEACS cohort. ${ }^{7}$

In their Heart publication, Rothnie et al report on the prognostic utility of COPD in a sample of 481849 patients with ACS admitted to UK hospitals in England and Wales and included in the myocardial ischaemia national audit project (MINAP) database. The authors compared the risk of death between patients with COPD and non-COPD at 6 months and they further assessed the incremental prognostic value when associated to GRACE risk score. COPD was present in $12.2 \%$ of patients with ACS and they had a 30\% higher risk of death, even after adjustment for GRACE risk score. As in previous observational studies, they have a particularly high-risk profile, including higher age, high prevalence of chronic heart failure, coronary artery disease, cerebrovascular disease, peripheral artery disease and chronic renal disease. Inclusion of COPD in the GRACE score equation, or multiplying the GRACE predicted risk of death by 1.3 for people with COPD were the two strategies proposed by the authors for improved outcome prediction. Approximately, one-third of patients with COPD categorised as low risk, are in fact moderate risk patients.

The association observed with all-cause mortality does not automatically mean that adding COPD as a new marker to a traditional risk prediction model will improve outcome risk prediction. Discriminative analysis of a model with GRACE risk score alone and after adjustment for COPD showed that c-statistic increased very slightly, although significantly. In the quantification of correct reclassification, COPD adjustment of a model with GRACE score was associated with an Net Reclassification Index (NRI) of $13 \%$, suggesting effective reclassification with similar results in different types of ACS. The magnitude of improvement was small, which was expected in the presence of a fairly robust risk score, such as in the case of GRACE risk score. In smaller sample populations, it might not be the case, because significance was attainable due to the very large sample.

The main strength of the present study is the very large sample size, obtained from a nationwide multicenter registry. The most important limitation is that COPD was diagnosed based on local physician assessment only. Pulmonary function tests were not routinely performed to diagnose and quantify COPD severity. Most studies on COPD and ACS used a clinical definition based in a previous history of COPD treated with pharmacological therapies for obstructive lung disease (inhaled steroids, inhaled anticholinergics, inhaled $\beta$-agonists theophylline) at hospital admission. This may lead to underestimation or misdiagnosis of COPD frequency. Previous studies reported very different prevalence's of COPD, ranging from $5.8 \%$ to $18.2 \%$ (table 1). A study with spirometry screening in patients with ACS obtained an objective COPD prevalence up to $11 \%$, 
Table 1 Reported prevalence of COPD in myocardial infarction patients

\begin{tabular}{llcl}
\hline Author & Country/study & Prevalence (\%) & Observations \\
\hline Salisbury et $a l^{12}$ & USA (PREMIER study) & 15.6 & Discharged alive \\
Dziewierz et $a l^{6}$ & Poland (registry) & 11.3 & \\
Hadi et $a l^{13}$ & Middle East (GULF RACE) & 5.3 & \\
Bursi et $a l^{14}$ & USA & 12.0 & \\
Stefan et $a l^{15}$ & USA & 17.0 & \\
Campo et $a l^{16}$ & Italy & 18.2 & \\
Andell et $a l^{17}$ & Sweden (Registry) & 6.0 & Discharged alive \\
Andell et $a l^{18}$ & International (PLATO trial) & 5.8 & \\
Pocock et al & International (EPICOR study) & 6.4 &
\end{tabular}

COPD, chronic obstructive pulmonary disease; EPICOR, tErm follow-up of antithrombotic management Patterns In acute CORonary syndrome patients; PLATO, platelet inhibition and patient outcome; PREMIER, prospective registry evaluating myocardial infarction: events and recovery.

when only $5 \%$ were positive by medical record review only. ${ }^{8}$ Another possible limitation for the results presented is related to data analysis. COPD and Killip class were not reported in MINAP database and the authors used surrogate markers to identify those patients. The National Institute for Health and Care Excellence amended mini-GRACE score was used instead. Although both methods were previously validated, nonetheless this is a cause for some possible bias.

However, one of the main questions is the real-benefit of including all those new prognostic variables in GRACE risk score. GRACE risk score nomogram includes several variables with a wide range of categories, adding some complexity to risk scoring. This might explain its underuse in clinical practice in spite of the development of several tools including internet-based calculators, also for mobile devices. ${ }^{10}$ Inclusion of other variables can increase complexity even further and compromise its use. Previous studies showed that it is possibly to simplify risk scores with simple and fewer variables, with similar predictive accuracy. The Canada Acute Coronary Syndrome risk score selected simple variables (age, Killip class, systolic blood pressure and heart rate) and it allows rapid stratification of patients with ACS including by healthcare professionals without advanced medical training at the prehospital setting or emergency department. ${ }^{11}$ It showed a good predictive value both for short-term and long-term mortality and in both STEMI and NSTEACS, although slightly lower when compared with classical risk scores. Maybe a strategy of early and simple risk stratification would be more appropriate with subsequent stratification considering all additional prognostic comorbidities identified so far, such as diabetes, renal function and COPD. In STEMI immediate reperfusion therapy is essential and risk stratification is less important. In NSTEACS, invasive treatment has a wide range of option, between immediate and 72 hours delay. Immediate invasive treatment can be selected by very simple clinical assessment and it does not require risk scoring. For other strategies, we have more time to consider the prognostic impact of other comorbidities. It is thus necessary to understand in real-life clinical practice, what is the real-impact of risk scoring, both in terms of use and in terms of management implications.

\section{Competing interests None declared.}

Provenance and peer review Commissioned; internally peer reviewed.

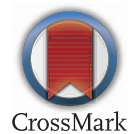

To cite Timóteo AT. Heart 2016:102:1423-1424.

Published Online First 10 June 2016

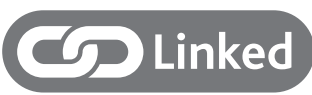

- http://dx.doi.org/10.1136/heartjnl-2016-309359

Heart 2016;102:1423-1424.

doi:10.1136/heartjnl-2016-309860

\section{REFERENCES}

1 Morrow DA, Antman EM, Charlesworth A, et al. TIMI risk score for ST-elevation myocardial infarction: a convenient, bedside, clinical score for risk assessment at presentation: an intravenous nPA for treatment of infarcting myocardium early II trial substudy. Circulation 2000;102:2031-7.

2 Antman EM, Cohen M, Bernink PJ, et al. The TIMI risk score for unstable angina/non-ST-elevation MI. A method for prognostication and therapeutic decision making. JAMA 2000:284:835-42.

3 Granger CB, Goldberg RJ, Dabbous O, et al. Predictors of hospital mortality in the Global Registry of Acute Coronary Events. Arch Intern Med 2003:163:2345-53.

4 Eagle KA, Lim MJ, Dabbous OH, et al. A validated prediction model for all forms of acute coronary syndrome: estimating the risk of 6-month postdischarge death in an international registry. J Am Med Assoc 2004;291:2727-33.

5 Rothnie KJ, Yan R, Smeeth L, et al. Risk of myocardial infarction (MI) and death following $\mathrm{Ml}$ in people with chronic obstructive pulmonary disease (COPD): a systematic review and meta-analysis. BMJ Open 2015:5:e007824.

6 Dziewierz A, Siudak Z, Rakowski T, et al. Relationship between chronic obstructive pulmonary disease and in-hospital management and outcomes in patients with acute myocardial infarction. Kardiol Pol 2010;68:294-301.

7 Pocock S, Bueno H, Licour M, et al. Predictors of one-year mortality at hospital discharge after acute coronary syndromes: a new risk score from the EPICOR (long-tErm follow-uP of antithrombotic management patterns In acute CORonary syndrome patients) study. Eur Heart I Acute Cardiovasc Care 2015;4:509-17.

8 Rothnie KJ, Smeeth L, Pearce N, et al. Predicting mortality after acute coronary syndromes in people with chronic obstructive pulmonary disease. Heart 2016:102:1442-8.

9 Mooe T, Stenfors N. The prevalence of COPD in individuals with acute coronary syndrome: a spirometry-based screening study. COPD 2015;12:453-61.

10 Yan AT, Yan RT, Huynh T, et al. Understanding physicians' risk stratification of acute coronary syndromes: insights from the Canadian ACS 2 Registry. Arch Intern Med 2009;169:372-8.

11 Huynh T, Kouz S, Yan AT, et al. Canada Acute Coronary Syndrome Risk Score: a new risk score for early prognostication in acute coronary syndromes. Am Heart J 2013:166:58-63.

12 Salisbury AC, Reid KJ, Spertus JA. Impact of chronic obstructive pulmonary disease on post-myocardial infarction outcomes. Am J Cardiol 2007;99:636-41.

13 Hadi HA, Zubaid M, Al Mahmeed W, et al. Prevalence and prognosis of chronic obstructive pulmonary disease among 8167 Middle Eastern patients with acute coronary syndrome. Clin Cardiol 2010:33:228-35.

14 Bursi F, Vassallo R, Weston SA, et al. Chronic obstructive pulmonary disease after myocardial infarction in the community. Am Heart $J$ 2010;160:95-101.

15 Stefan MS, Bannuru RR, Lessard D, et al. The impact of COPD on management and outcomes of patients hospitalized with acute myocardial infarction: a 10-year retrospective observational study. Chest 2012;141:1441-8.

16 Campo G, Guastaroba P, Marzocchi A, et al. Impact of COPD on long-term outcome after ST-segment elevation myocardial infarction receiving primary percutaneous coronary intervention. Chest 2013:144:750-7.

17 Andell P, Koul S, Martinsson A, et al. Impact of chronic obstructive pulmonary disease on morbidity and mortality after myocardial infarction. Open Heart 2014:1:e000002.

18 Andell P, James SK, Cannon CP, et al. Ticagrelor Versus Clopidogrel in Patients With Acute Coronary Syndromes and Chronic Obstructive Pulmonary Disease: An Analysis From the Platelet Inhibition and Patient Outcomes (PLATO) Trial. J Am Heart Assoc 2015:4:e002490 